\title{
Occurrence and Natural History of Thyroid Cancer in Patients with Cowden Syndrome
}

\author{
Thomas Szabo Yamashita Fady J. Baky Travis J. McKenzie \\ Geoffrey B. Thompson David R. Farley Melanie L. Lyden Benzon M. Dy \\ Department of Surgery, Mayo Clinic, Rochester, MN, USA
}

\author{
Keywords \\ Cowden syndrome - Differentiated thyroid cancer - PTEN \\ mutation - Thyroidectomy
}

\begin{abstract}
Introduction: Cowden syndrome patients are at an increased risk of developing differentiated thyroid carcinoma (DTC). There are limited studies of thyroid cancer biology within this population. We aimed to describe the frequency and progression of DTC in this population. Method: We reviewed all patients with clinically or genetically confirmed Cowden syndrome treated at our institution between 1979 and 2017. Presentation, operative details, pathology, and adjuvant treatment for all thyroid diseases were analyzed. Results: Forty-seven patients were identified. Thirty-seven received a clinical diagnosis while 10 tested positive on genetic testing for the PTEN gene. Average follow-up time was 10 years. Twenty-three patients underwent a total thyroidectomy. Nine patients were diagnosed with thyroid cancer, with a mean age of 28.5 years, 4 cases of the follicular variant of papillary thyroid cancer (PTC), 4 of PTC, and 1 of cystic PTC. One patient required an interval lateral neck dissection but no patients developed distant metastasis. Fourteen patients
\end{abstract}

underwent thyroidectomy, 12 due to follicular adenoma, Hürthle cell adenoma, or multinodular goiter, 1 due to Graves' disease, and 1 who elected to undergo a prophylactic thyroidectomy. No mortality was associated with thyroid cancer in these patients. Conclusion: Patients with Cowden syndrome are at a significantly increased risk of developing DTC and have a younger age of onset. In this study, $20 \%$ of the patients with Cowden syndrome were diagnosed with DTC, but distant metastasis and overall mortality were absent. Clinical features were similar to those of sporadic DTC, proving that, apart from screening, Cowden syndrome-related DTC can be treated in similar fashion.

\footnotetext{
(c) 2020 European Thyroid Association Published by S. Karger AG, Basel
}

\section{Introduction}

Cowden syndrome (multiple hamartoma syndrome) is an autosomal dominant, inherited cancer syndrome associated with a mutation in the PTEN tumor suppressor gene. While Cowden syndrome is the most common inherited PTEN mutation, it remains rare with an estimated incidence of 1 in 200,000-250,000 [1,2]. As such, providers can be unfamiliar with these patients and the sur-

$\begin{array}{ll}\text { karger@karger.com } & \text { @ 2020 European Thyroid Association } \\ \text { www.karger.com/etj } & \text { Published by S. Karger AG, Basel } \\ \text { Karger } & \end{array}$


gical management of the disease. Features of Cowden syndrome are benign and malignant mucocutaneous changes including trichilemmomas, acral keratosis, gastrointestinal polyposis, and papillomatous lesions as well as an increased risk of thyroid, breast, and endometrial neoplasms $[1,3]$. Historically, Cowden syndrome was diagnosed on the basis of such clinical features as well as on an early presentation of thyroid or breast cancer. Efforts by the International Cowden Consortium in the late 1990s and early 2000s helped localize causative mutations and ultimately identify the affected gene, PTEN. As genetic screening for the disease has improved, patients are increasingly aware of their mutation at an early age, prior to the development of malignancy. It is important to note, however, that only approximately $80 \%$ of patients with Cowden syndrome will have a mutation localized to PTEN and, as a result, clinical presentation should continue to guide diagnosis and adherence to screening recommendations.

Currently, the National Comprehensive Cancer Network (NCCN) suggests regimented screening for breast, uterine, and thyroid cancers as well as renal ultrasound and colonoscopy. Thyroid ultrasound should begin at the age of 18 years and be repeated annually $[4,5]$. Increased adherence to these screening guidelines has resulted in increased presentation of Cowden syndrome patients to general and endocrine surgeons.

Multiple mutations are known to be associated with papillary thyroid cancer, including the RET/PTC and $B R A F$ mutations $[5,6]$. Cowden syndrome-associated malignancies occur secondary to PTEN gene mutations. Literature describing the incidence, natural course, and clinical aspects of PTEN-related thyroid cancer are sparse. This retrospective, single-institution study sought to analyze the mentioned aspects of PTEN-related thyroid cancer.

\section{Materials and Methods}

All patients with the diagnosis of Cowden syndrome and who were treated at Mayo Clinic between January 1979 and December 2017 were included in this single-institution, retrospective review. Information regarding clinical presentation, diagnosis, pathology, surgical treatment, follow-up, and surveillance for all thyroid neoplasia was analyzed. Pathology slides and imaging were not reinterpreted from outside institutions for the purpose of the study; analysis was based on reports available in medical records. No minimal follow-up duration was determined for the inclusion of patients. Descriptive statistics are used to report categorical data. Continuous data are reported as mean and standard deviation. All statistical analysis was performed using JMP v14 (Carey, NC, USA).

\section{Results}

There were 47 patients treated at our institution with a diagnosis of Cowden syndrome between 1979 and 2017. Median length of follow-up was 10 years (0-24 years). There were 37 patients who were diagnosed based on clinical features and family history, while 10 had genetic testing that indicated a mutation of the PTEN gene. Mutations found in our cohort included: c.388C $>\mathrm{T}$, c. $634+5 \mathrm{G}$, c. $900 \mathrm{del}$, c. $920 \mathrm{G}>\mathrm{A}$, c. $737 \mathrm{C}>\mathrm{Y}$, c. $464 \mathrm{~A}>\mathrm{G}$, and $c .414 \mathrm{~T}>\mathrm{G}$. Three patients underwent genetic testing in addition to clinical diagnosis. For the 38 patients who received their diagnosis of Cowden syndrome at our institution, the mean age at diagnosis was 45 years (15-74 years). Patients who were diagnosed based on clinical presentation alone had a mean age at diagnosis of 47 years versus 41 years if genetic testing was performed. All patients who did not undergo genetic testing met satisfactory clinical criteria for Cowden syndrome at the time of diagnosis. The most recent patients in our series were diagnosed based on the NCCN guidelines; 35\% were diagnosed based on pathognomonic criteria, $35 \%$ on the presence of at least 2 major criteria, $20 \%$ on the presence of 1 major and $>3$ minor criteria, and $10 \%$ had $>4$ minor criteria. The most prevalent major clinical feature was breast cancer (29\%), followed by thyroid cancer (24\%), endometrial cancer (15\%), and macrocephaly (3\%). Amongst the minor clinical criteria, benign thyroid abnormalities were the most common (26\%), followed by gastrointestinal hamartomatous polyps (21\%), uterine fibroids (15\%), benign fibrocystic disease of the breast (12\%), genitourinary malformations (9\%), fibromas (6\%), and intellectual disability (6\%).

A total of $22(47 \%)$ patients underwent a total thyroidectomy, one being a completion thyroidectomy after lobectomy in outside institution. One patient underwent lobectomy prior to the diagnosis of Cowden syndrome without further surgery. Among patients in the surgical cohort, $57 \%$ were initially evaluated due to clinically significant goiter, $21 \%$ due to solitary thyroid nodules, $7 \%$ due to imaging findings under surveillance, and $14 \%$ were found to have suspicious nodules during imaging performed for other causes. Preoperative ultrasound showed that $83 \%$ of patients who underwent thyroidectomy had multinodular goiter, with a minority presenting solitary nodules. There were 9 (19\%) patients diagnosed with thyroid cancer at a mean age of 28.5 years ( $16-41$ years) and all underwent total thyroidectomy. Four of nine patients (44\%) reported a follicular variant of papillary thyroid cancer (PTC), 1 had cystic PTC (12\%), and 4 had conven- 
tional PTC (44\%). Five patients had a preoperative diagnosis of cervical nodal metastasis. The most common findings in patients with benign pathology were: multinodular goiter $(43 \%)$, follicular adenoma $(36 \%)$, normal thyroid (7\%), lymphocytic thyroiditis (7\%) and colloid cyst $(7 \%)$.

Seven patients who underwent a total thyroidectomy due to thyroid cancer were diagnosed with Cowden syndrome after undergoing thyroidectomy. All of them presented with incidentally noted thyroid masses. The other 2 patients from the cohort were diagnosed with thyroid cancer after surveillance ultrasound. Mean tumor size was $1.8 \mathrm{~cm}(0.5-5.0 \mathrm{~cm})$ and only 1 patient presented with microcarcinoma. One patient had 2 distinct, bilateral tumors. One patient required an interval lateral neck dissection, but no patients developed distant metastatic disease. Of the 38 patients who were not diagnosed with thyroid cancer, an additional 14 (37\%) underwent thyroidectomy, 12 due to follicular adenoma, Hürthle cell adenoma, or multinodular goiter, 1 due to Graves' disease, and 1 who elected to undergo a prophylactic thyroidectomy. No mortality was associated with thyroid cancer in any patients.

Twenty-four patients did not undergo thyroidectomy. Thirty-three percent were followed with ultrasound screening at our institution and $25 \%$ did not have ultrasound. One patient was followed by physical exam, 2 patients had ultrasound surveillance recommended but the imaging was not performed, and 9 patients were lost to follow-up. Among the patients who were appropriately screened, none developed thyroid cancer during followup.

\section{Discussion}

Patients with Cowden syndrome are at a significantly increased risk of developing DTC at a younger age of onset $[1,3,4,7-10]$. As such, early screening often results in presentation to an endocrine surgeon for evaluation of thyroid masses discovered on screening ultrasounds.

In total, 13 patients underwent genetic testing and PTEN mutations were found in $77 \%$. One patient had an indeterminate result which was not repeated, while 2 were not found to have any mutations in the PTEN gene after repeat genetic testing. Both of these patients continued to carry a diagnosis of Cowden syndrome due to the clinical criteria and continued screening was recommended. Our rate of $77 \%$ positive gene localization is consistent with estimates of an $80 \%$ correlation between

Thyroid Cancer in Patients with Cowden Syndrome clinical Cowden syndrome and a localized PTEN mutation.

Previous studies have estimated the lifetime risk of thyroid cancer in patients with Cowden syndrome to be $35 \%[7-9,11]$. At our institution, all thyroid cancers associated with Cowden syndrome were a variant of PTC. This is in contrast to the literature which suggests a higher incidence of follicular cancer. Almost half of this population was diagnosed with the follicular variant of PTC. Pathology specimens were not reviewed and we collected our data from the available medical reports. We hypothesize that this, combined with the tertiary referral center nature of our institution and the long time-frame could have led to this discrepancy. Patients in our cohort had a mean age of onset of 28.5 years, significantly earlier than in the general population which has a mean age of onset of 49 years. Five patients had positive cervical lymph nodes, which is higher than previously described. This must be interpreted carefully in the context of the limited sample size [11]. No mortality or distant metastasis was reported in this study.

Rates of thyroidectomy exceeded rates of thyroid malignancy in our study population, with nearly $50 \%$ of the cohort undergoing thyroidectomy. Almost one third of the individuals in our cohort underwent thyroidectomy for benign thyroid disease. Seven patients underwent thyroidectomy after evaluation of a follicular adenoma without evidence of malignancy on FNA. Six patients underwent thyroidectomy with a diagnosis of multinodular goiter and 1 elected to undergo prophylactic thyroidectomy without evidence of disease at the age of 42 years.

As a retrospective study, there are limitations to this case series. Because of the wide range of time, multiple medical records systems were used, creating an unavoidable risk of data loss or misinterpretation. As a tertiary center, many patients were referred from other centers and were partially treated and followed up in other institutions, making data collection and interpretation harder. Multiple details, including pathology and imaging variables, were abstracted from reports available only from the medical records.

In 2012, Milas et al. [4] raised the question of prophylactic thyroidectomy in certain patients with Cowden syndrome, particularly in those with developmental delay or who may struggle to adhere to screening guidelines. Our study reiterated the knowledge that Cowden syndrome patients have a higher incidence of thyroid cancer and at an earlier age. However, there was no mortality or distant metastasis from thyroid cancer in our patients, 
and of the patients that did not undergo thyroidectomy, none developed thyroid cancer during follow-up. This shows that even though Cowden syndrome is a PTENrelated disease, it does not appear to be clinically more aggressive or have a different natural history than other forms of DTC. Surveillance with neck ultrasound is a valuable tool, and in the case of suspicious findings, diagnostic and treatment algorithms can follow the already established pathways taken for detecting other sporadic forms of DTC.

\section{Statement of Ethics}

Appropriate institutional review board approval for all human investigations was obtained. In addition, for investigations involving human subjects, informed consent was obtained from the participants.

\section{Disclosure Statement}

There was no proprietary or commercial interest in any product mentioned or concept discussed in this article.

\section{Funding Sources}

This research was conducted with the authors' discretionary funds only.

\section{Author Contributions}

Project conceived and analysis design by Benzon Dy. Data collection by Fady Baky. Data analysis, review, and writing by Fady Baky and Thomas Szabo Yamashita. Revision and final approval of the version to be published by Travis McKenzie, Geoffrey Thompson, David Farley, Melanie Lyden, and Benzon Dy.

\section{References}

1 Tan MH, Mester JL, Ngeow J, Rybicki LA, Orloff MS, Eng C. Lifetime cancer risks in individuals with germline PTEN mutations. Clin Cancer Res. 2012 Jan;18(2):400-7.

2 Kameyama K, Takami H, Miyajima K, Mimura T, Hosoda Y, Ito K, et al. Papillary carcinoma occurring within an adenomatous goiter of the thyroid gland in Cowden's disease. Endocr Pathol. 2001;12(1):73-6.

3 Pilarski R, Eng C. Will the real Cowden syndrome please stand up (again)? Expanding mutational and clinical spectra of the PTEN hamartoma tumour syndrome. J Med Genet. 2004 May;41(5):323-6.

4 Milas M, Mester J, Metzger R, Shin J, Mitchell J, Berber E, et al. Should patients with Cowden syndrome undergo prophylactic thyroidectomy? Surgery. 2012 Dec;152(6):1201-10.
5 Haugen BR. 2015 American Thyroid Association Management Guidelines for Adult $\mathrm{Pa}$ tients with Thyroid Nodules and Differentiated Thyroid Cancer: what is new and what has changed? Cancer. 2017 Feb;123(3):37281.

6 Kimura ET, Nikiforova MN, Zhu Z, KnaufJA, Nikiforov YE, Fagin JA. High prevalence of BRAF mutations in thyroid cancer: genetic evidence for constitutive activation of the RET/PTC-RAS-BRAF signaling pathway in papillary thyroid carcinoma. Cancer Res. 2003 Apr;63(7):1454-7.

7 Laury AR, Bongiovanni M, Tille JC, Kozakewich $\mathrm{H}$, Nosé V. Thyroid pathology in PTENhamartoma tumor syndrome: characteristic findings of a distinct entity. Thyroid. 2011 Feb;21(2):135-44.

8 Hall JE, Abdollahian DJ, Sinard RJ. Thyroid disease associated with Cowden syndrome: A meta-analysis. Head Neck. 2013 Aug;35(8): 1189-94.
9 Ngeow J, Mester J, Rybicki LA, Ni Y, Milas M, Eng C. Incidence and clinical characteristics of thyroid cancer in prospective series of individuals with Cowden and Cowden-like syndrome characterized by germline PTEN, SDH, or KLLN alterations. J Clin Endocrinol Metab. 2011 Dec;96(12):E2063-71.

10 Cameselle-Teijeiro J, Fachal C, Cabezas-Agrícola JM, Alfonsín-Barreiro N, Abdulkader I, Vega-Gliemmo A, et al. Thyroid Pathology Findings in Cowden Syndrome: A Clue for the Diagnosis of the PTEN Hamartoma Tumor Syndrome. Am J Clin Pathol. 2015 Aug; 144(2):322-8.

11 Grebe SK, Hay ID. Thyroid cancer nodal metastases: biologic significance and therapeutic considerations. Surg Oncol Clin N Am. 1996 Jan;5(1):43-63. 